\title{
OPTIMALITY CRITERIA FOR THE PREDICTION OF METABOLIC FLUXES IN YEAST MUTANTS
}

\author{
EVAN S. SNITKIN ${ }^{1}$ \\ esnitkin@bu.edu
}

\author{
DANIEL SEGRE $\dot{1}^{1,2}$ \\ dsegre@bu.edu
}

\begin{abstract}
'Graduate Program in Bioinformatics, Boston University, 44 Cummington St., Boston, Massachusetts, 02215, USA

${ }^{2}$ Departments of Biology and Biomedical Engineering, Boston University, 24 Cummington St., Boston, Massachusetts, 02215, USA
\end{abstract}

\begin{abstract}
Constraint-based models of cellular metabolism, such as flux balance analysis (FBA), use convex analysis and optimization to study metabolic networks at a genome scale. The availability of reaction lists for numerous organisms, along with a variety of network analysis and optimization tools, is making these approaches increasingly popular for metabolic engineering and biomedical applications, as well as for addressing fundamental biological questions. It is therefore very important to assess the predictive capacity of these models and to understand how to interpret them in a biologically relevant manner. Typically, model assessment is limited to gauging the ability to predict phenotypes, such as viability under different environmental and genetic conditions. These types of assessments, for the most part, focus only on the growth phenotype of the cells, but ignore the underlying flux predictions. While this may be sufficient for certain types of study, the question of whether flux balance models can reliably predict intracellular and transport fluxes is crucial for more detailed analysis, and remains largely unanswered. Here we compare FBA model predictions of yeast metabolic fluxes to a previously published set of experimentally determined fluxes for 13 different single gene deletion mutants across a variety of possible objective functions. We find that the specific optimization criteria used to determine fluxes have a significant impact on the accuracy of the predicted fluxes. Interestingly, while different optimization methods provide very different levels of agreement relative to experimental fluxes, they tend to provide similar predictions with respect to the effect of the perturbation on growth. This demonstrates that assessment of models at the level of flux predictions is a critical step in assessing the biological validity of different models and optimization criteria.
\end{abstract}

Keywords: flux balance analysis; gene deletion; optimality criteria; flux measurements

\section{Introduction}

A century of detailed biochemical studies, in conjunction with the genomic revolution, has culminated in the release of metabolic reconstructions for a number of model organisms. These metabolic reconstructions comprise the stoichiometries of all known enzymatic reactions in a given organism. In addition to enabling the study of metabolic networks in diverse organisms [19], these reconstructions have yielded the ability to create genome-scale predictive models by using the steady state framework of flux balance analysis [12]. Flux balance models have been released for a number of bacterial organisms such as E. coli [7] and H. pylori [14], and more recently also for the eukaryotes yeast [9] and human [5]. With the ability to generate models largely from sequence data, it should be expected that the pace of model development will only increase in the coming months and years. 
Along with the increase in model availability has come a widening of the spectrum of reported applications of flux balance models. Recent work has demonstrated the use of flux balance models to address cutting edge research questions ranging from understanding the dynamics of microbial communities [17] to predicting perturbations required to fulfill complex metabolic engineering objectives [2]. These various applications of flux balance models often require different levels of predictive abilities from the models. For instance, for some applications, being able to accurately capture the range of possible metabolic behaviors of an organism is sufficient [3], while for others the ability to predict the precise metabolic state resulting from specific perturbations is required [2].

Given that different model applications may require different levels of predictive proficiency, it is important to be able to evaluate the appropriateness of models for addressing different research questions. A common method for evaluating models is by quantifying their abilities to predict the effects of environmental and genetic perturbations on growth rate. The attractiveness of this approach for model evaluation largely stems from the availability of high-throughput growth phenotype data for many organisms, in addition to the ease with which the effects of environmental and genetic perturbations on growth can be determined using these models. While such assessments evaluate model behavior in response to diverse perturbations, the assessments are typically limited to growth phenotype. An open question is how a model's ability to predict the growth phenotypes under a variety of conditions translates into its ability to predict the fluxes underlying the growth predictions.

Here, we utilized a compendium of experimentally determined fluxes for yeast single gene deletion mutants [1] to gain insight into the ability of yeast flux balance models to predict central carbon metabolic fluxes in response to perturbations. In addition to assessing the relationship between predictions of growth phenotypes and predictions of the underlying fluxes, we also compared the ability of different objective functions to predict the metabolic response to genetic perturbations. Through this analysis we hoped not only to assess the predictive abilities of flux balance models at the level of flux predictions, but also to understand what drives the metabolic response to genetic perturbations. Our results support previous studies which suggested that the metabolic response to genetic perturbations is best described as a minimal rerouting of fluxes around the perturbation. Despite the clear superiority of an objective function implementing minimal flux rerouting to predict mutant fluxes, all tested objective functions correctly predicted the growth phenotype for all 13 mutants considered. This suggests that correct predictions of growth phenotype do not necessarily imply an accurate prediction of the underlying fluxes.

\section{Methods}

\subsection{Experimental flux data}

All experimentally measured fluxes and uptake/secretion rates were taken from the supplementary material of the 2005 manuscript by Blank et al. [1]. Among the 38 single gene deletion mutants for which fluxes were measured, we focused on 13 for which the deleted gene did not have any duplicates. The reason for this is that gene duplicates are 
implemented in a trivial manner in flux balance models, unless regulation is explicitly taken into account. In a typical flux balance calculation duplicate genes completely back one another up under all conditions.

\subsection{Flux Balance Analysis}

Flux balance analysis is a linear constraint based modeling approach which has been described in detail elsewhere [6]. Briefly, flux balance analysis consists of two critical steps; (1) the imposition of linear constraints on fluxes, stemming from the assumption of steady state, and (2) an optimization step by which a particular set of fluxes fulfilling the given constraints is selected. These linear constraints limit the feasible flux solutions to those which result in no net production or consumption of any metabolite. These steady state constraints can be described by the nullspace of the $m \times n$ stoichiometric matrix $\mathrm{S}$. The columns of $S$ represent the $n$ reactions, and its rows the $m$ different metabolites. An entry $S_{i j}$ represents the stoichiometric coefficient of metabolite $i$ in reaction $j$. In addition to the steady state constraints, additional linear constraints are imposed to set upper and lower bounds on individual fluxes $\left(a_{j} \leq v_{j} \leq b_{j}\right)$. These constraints can be applied to fix maintenance requirements, restrict reversibility of reactions and set limits on nutrient uptake rates.

The previously released iLL672 yeast metabolic reconstruction was used for all analyses [13]. Constraints on uptake rates were imposed to mimic the minimal glucose conditions under which the utilized set of experimentally determined fluxes were determined. Gene deletions were implemented in the model by setting the flux to zero for all reactions requiring the protein product of the deleted gene.

\subsection{Objective functions to predict mutant fluxes}

While the imposition of the linear constraints mentioned above restricts the space of possible metabolic behaviors, there are still potentially an infinite number of flux states which can fulfill the given constraints. To select a particular flux state, which can in turn be compared to the experimentally measured fluxes, one typically maximizes or minimizes a linear combination of fluxes, based on a biologically relevant criterion. Here we evaluated the flux predictions made using several different criteria. A summary of the different objective functions and the motivation for testing them can be found in Table 1.

\section{Results}

\subsection{Experimentally determined fluxes}

To evaluate the relative abilities of different objective functions to accurately predict the metabolic flux response to genetic perturbations, we utilized the aforementioned compendium of experimentally determined fluxes for $S$. cerevisiae single gene deletion mutants [1]. The mutants analyzed by Blank et al. were selected on the basis that the deleted genes encoded enzymes which catalyzed reactions that were active under minimal glucose conditions, but were not essential to growth. In other words, these genes encoded enzymes in flexible reactions, such that by observing how the metabolic network responds to their deletion, insight could be gained into the metabolic basis for 
the robustness to gene deletions that has been previously observed in yeast metabolism [ 1 , 4].

Despite the fact that the set of mutants analyzed by Blank et al. targeted genes in various central carbon metabolic processes, the nature of the metabolic flux responses were largely similar. Specifically, it was observed that for most mutants, the metabolic response was a local rerouting of flux around the perturbed reaction, with the relative flux through other pathways remaining similar to the wildtype. The exceptions to this rule were for mutants in reactions critical to redox metabolism, where more distant rerouting was observed. An important caveat to the observed similarity in the flux distributions of the different mutants is that the absolute flux of carbon varied greatly. This aspect of the deletion mutant response is demonstrated in Fig. 1, where the glucose uptake and biomass production for the 13 mutants analyzed in the current study are shown. It can be seen that although the efficiency with which carbon is utilized is largely similar across different mutants, the growth rates vary greatly.

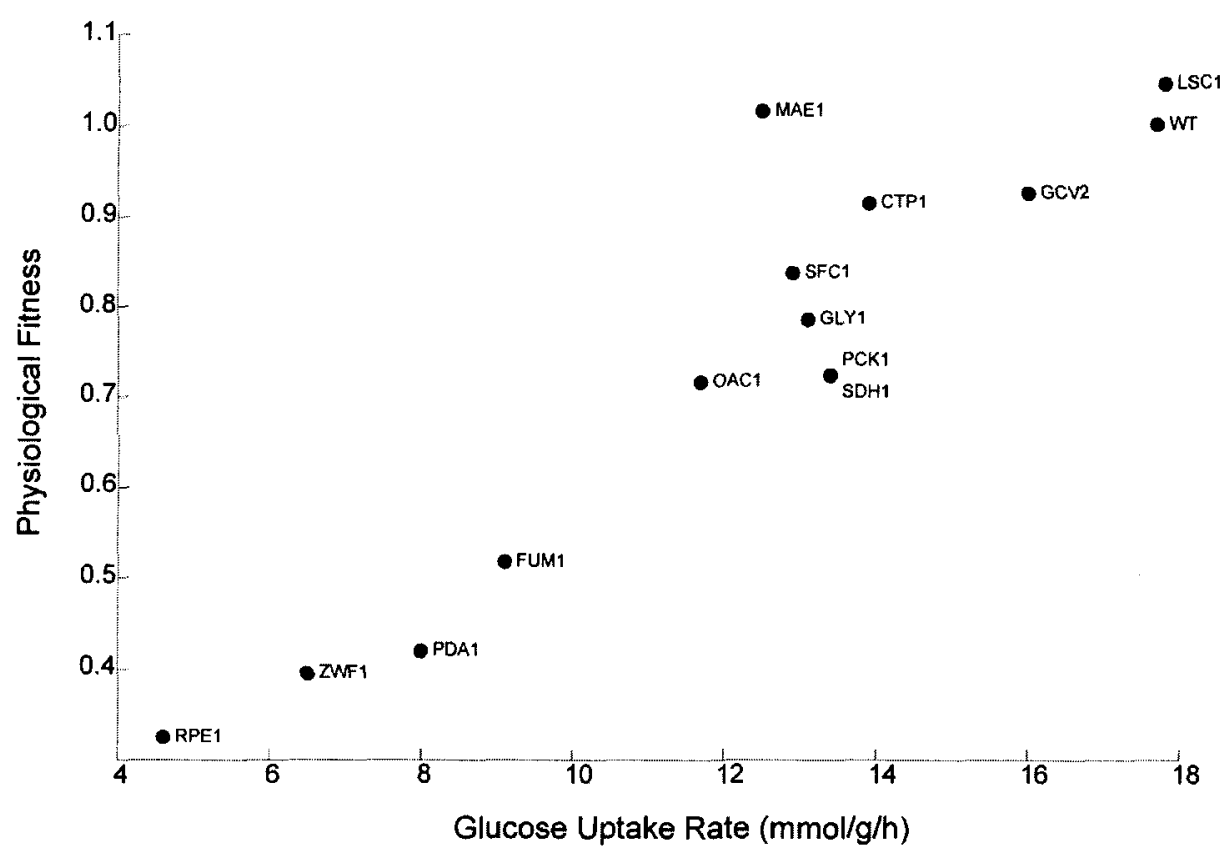

Fig. 1. Experimentally determined glucose uptake rates and fitness for strains analyzed in current study.

Glucose uptake rates were plotted against the physiological fitness for the 13 mutants analyzed in the current study, along with the wildtype. Each point represents an individual strain, which is labeled with the gene which was deleted, or with WT if no gene was deleted. Physiological fitness was computed by normalizing a strains growth rate by that of the wildtype. The wide range of glucose uptake rates indicates variation in the absolute metabolic flux carried in the different mutants. On the other hand, the strong correlation between glucose uptake rate and physiological fitness suggests that the glucose is largely being used in a similar manner across the different mutants. 


\subsection{Objective functions used to predict mutant fluxes}

Our assessment of the ability of yeast flux balance models to predict fluxes in single gene deletion mutants included the evaluation of a set of 9 different objective functions (See Table 1). These 9 objective functions can be dissected into four categories: growth maximization, minimization of metabolic adjustment, experimentally motivated and alternate maximization criteria.

Table 1. Objective functions used to determine mutant fluxes.

\begin{tabular}{|c|c|c|}
\hline Optimization Method & $\begin{array}{c}\text { Primary Optimization } \\
\text { Function }\end{array}$ & Additional Notes \\
\hline FBA_MIN_AV & $\max v_{g r o w t h}^{K O}$ & $\begin{array}{l}\text { A secondary optimization was } \\
\text { performed to minimize the sum of the } \\
\text { absolute values of the fluxes }\end{array}$ \\
\hline FBA_WT_MIN_DIST & $\max v_{g r o w t h}^{K O}$ & $\begin{array}{l}\text { A secondary optimization was } \\
\text { performed to minimize the distance } \\
\text { from an experimentally constrained } \\
\text { WT solution }\end{array}$ \\
\hline MOMA_LP & $\sum_{i}\left|v_{i}^{K O}-v_{i}^{W T}\right|$ & $\begin{array}{l}\text { LP refers to the use of linear } \\
\text { programming to minimize the } \\
\text { Manhattan distance }\end{array}$ \\
\hline MOMA_QP & $\left(v_{i}^{K O}-v_{i}^{W T}\right)^{2}$ & $\begin{array}{l}\text { QP refers to the use of quadratic } \\
\text { programming to minimize Euclidean } \\
\text { distance }\end{array}$ \\
\hline MOMA_LP_WT_CONSTR & $i_{i}^{K O}-v_{i}^{W T} E X P$ & $\begin{array}{l}\text { The experimentally constrained WT } \\
\text { solution was computed minimizing } \\
\text { the sum of fluxes, given the } \\
\text { experimental constraints [13]. }\end{array}$ \\
\hline MOMA_QP_WT_CONSTR & $\min \sum_{i=1}^{m}$ & \\
\hline MOMA_LP_GLC_UP_NORM & $\min \sum_{i=1}^{m} \mid \frac{v_{i}^{K O}}{v_{G L C}^{K O}}-\frac{v_{i}^{W T}}{v_{G L C}^{W T}}$ & \\
\hline MOMA_LP_BM_SINK & $\min \sum_{i=1}^{m}\left|v_{i}^{K O}-v_{i}^{W T}\right|$ & $\begin{array}{l}\text { During the optimization sink } \\
\text { reactions were created for each } \\
\text { biomass component }\end{array}$ \\
\hline FBA_MAX_ETOH & $\max v_{E t O H}^{K O}$ & $\begin{array}{l}\text { For both primary and secondary } \\
\text { optimizations biomass was fixed to } \\
\text { the experimental value determined } \\
\text { for the given mutant }\end{array}$ \\
\hline Abbreviations: $\mathrm{WT}=$ Wildt & $\begin{array}{l}\text { Knock Out LP }=\text { Linear Progra } \\
\text { lass, } G L C=G \text { Glucose, } E X P=E \text { ? }\end{array}$ & $\begin{array}{l}\mathrm{QP}=\text { Quadratic Programming, } \\
\text { ntal }\end{array}$ \\
\hline
\end{tabular}

\subsubsection{Growth maximization}

This set consisted of two objective functions, which both select flux solutions which maximize biomass production. The two objective functions differ in their secondary objective functions, which are used to select among the set of alternative flux solutions which all result in optimal biomass production. The first, FBA_MIN_AV, performs a 
secondary optimization which finds the flux distribution which produces the optimal biomass and has the minimal sum of the absolute values of fluxes through all reactions. The hypothesis underlying this approach is that yeast will attempt to achieve maximal growth at a minimal expense in terms of enzyme usage $[10,15]$. The second objective function, FBA_WT_MIN_DIST, performs a secondary optimization which finds the set of fluxes which produces the optimal biomass and has the minimal Manhattan distance from an experimentally constrained wildtype solution. The motivation for this secondary objective was the aforementioned observation that the distribution of flux in deletion mutants is overall very similar to the wildtype.

\subsubsection{Minimization of metabolic adjustment}

This set consisted of four objective functions all of which minimize the distance from a wildtype flux solution, given the additional constraint of the gene deletion [16]. These objectives differ in the distance metric used and the wildtype flux solution to which the distance was minimized. The distance metrics were Manhattan (MOMA_LP and MOMA_LP_WT_CONSTR) and Euclidean (MOMA_QP ${ }^{-}$and MOMA_QP_WT_CONSTR) distances, both of which have been used in previous applications of the minimization of metabolic adjustment criteria $[13,16]$. The wildtype flux distributions differed in that one uses experimental flux data to constrain the solution space (MOMA_LP_WT_CONSTR and MOMA_QP_WT_CONSTR), and the other does not (MOMA_LP and MOMA_QP).

\subsubsection{Experimentally motivated}

Both of the experimentally motivated objective functions are derivatives of minimization of metabolic adjustment, but with additions which were motivated by some of the observations made by Blank et al. [1], and others [8], in the analysis of fluxes in genetic mutants. MOMA_GLC_NORM used an experimentally constrained wildtype solution as above, but minimized the distance between fluxes normalized by the glucose uptake rate (See Table 1). The motivation for MOMA_GLC_NORM was the observed variation in the absolute flux among the different deletion mutants. The second objective is MOMA_BM_SINK, which minimized the Manhattan distance from an experimentally constrained wildtype solution as above, but included sink reactions for all biomass components. The motivation for MOMA_BM_SINK was to alleviate constraints on maintaining wildtype growth, when minimizing distance to the wildtype flux solution.

\subsubsection{Alternate maximization criteria}

The only objective function in this category maximized ethanol production in the mutant, given that biomass production was fixed to the experimentally observed value. The FBA_MAX_ETOH objective was motivated by the well known phenomenon whereby yeast preferentially ferments glucose, although it can be more efficiently broken down through oxidative phosphorylation [11]. Some have theorized that this aspect of yeast metabolism is a result of a selective advantage in maximizing ethanol production, so as to create a poor environment for potential competitors [18]. 


\subsection{Correlations between experimental and predicted fluxes}

Initial evaluation of the different objective functions was done by computing the Spearman Rank correlation between predicted fluxes and 36 experimental flux measurements. These 36 fluxes, which consist of fluxes through central carbon metabolism along with uptake/secretion rates, were selected for correlation analysis because they represent a set of linearly independent variables in the genome scale yeast model used. The results of the correlation analysis are shown in Fig. 2 for four optimization methods, which were found to be representative of the nine evaluated. For all 13 mutants tested, the objective functions which computed minimal distance from an experimentally determined wildtype solution achieved the best correlations. The performance of this set of methods was largely unaffected by the choice of distance metric (Manhattan or Euclidean), the addition of sinks for biomass components or by computing distances based on fluxes normalized by glucose uptake rates. On the other hand, the nature of the wildtype reference from which the distance was minimized was found to be very important. Specifically, inferior performance was observed across all mutants when using the method which minimizes the distance from a wildtype solution predicted by assuming maximal biomass production.

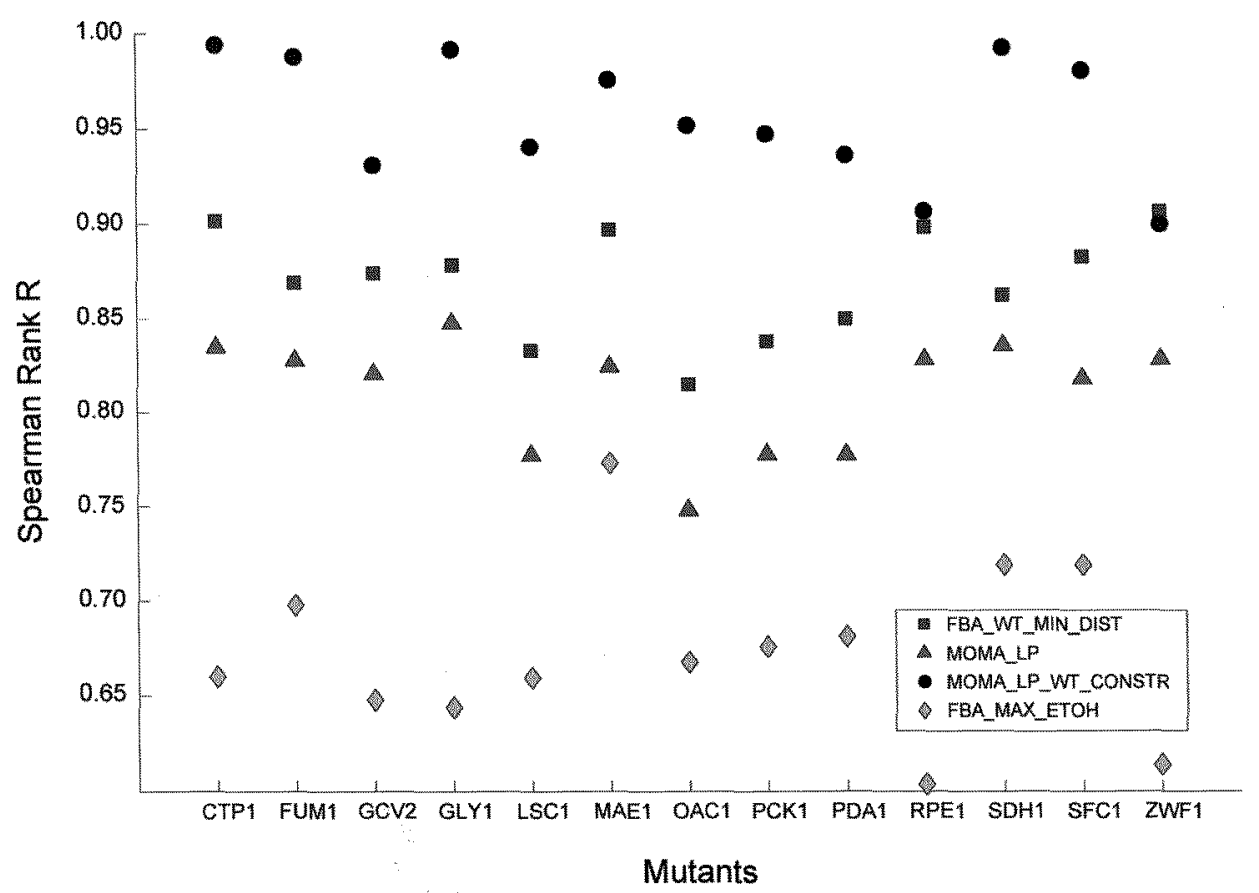

Fig. 2. Spearman correlations of predicted fluxes with experimentally determined fluxes.

Spearman rank correlation $\mathrm{R}$ values were computed between experimentally determined fluxes and the fuxes predicted by each of the 9 objective functions for the 13 different gene deletion mutants. Here, the $R$ values for 4 objective functions are shown, as these 4 were found to be representative of all 9. Specifically, MOMA LP performed the same as MOMA QP, while MOMA LP_WT_CONSTR performed the same as MOMA_QP WT_CONSTR, MOMA_GLC NORM, and MOMA_BM SNK. For virtually all mutants the strongest correlation was achieved using an objective which minimized the distance from an experimentally 
constrained wildtype flux solution (black circles). The reference flux solution was critical, as minimizing the distance from a wildtype solution computed with the assumption of optimal growth resulted in a decreased correlation in all mutants (gray triangles). The objective maximizing production of ethanol (gray diamonds), produced fluxes which were least correlated with the experimental measurements. Notably, despite the respirofermentative behavior of yeast in aerobic glucose conditions, maximization of ethanol did a worse job of describing the flux response than maximization of growth (black squares) for all 13 mutants.

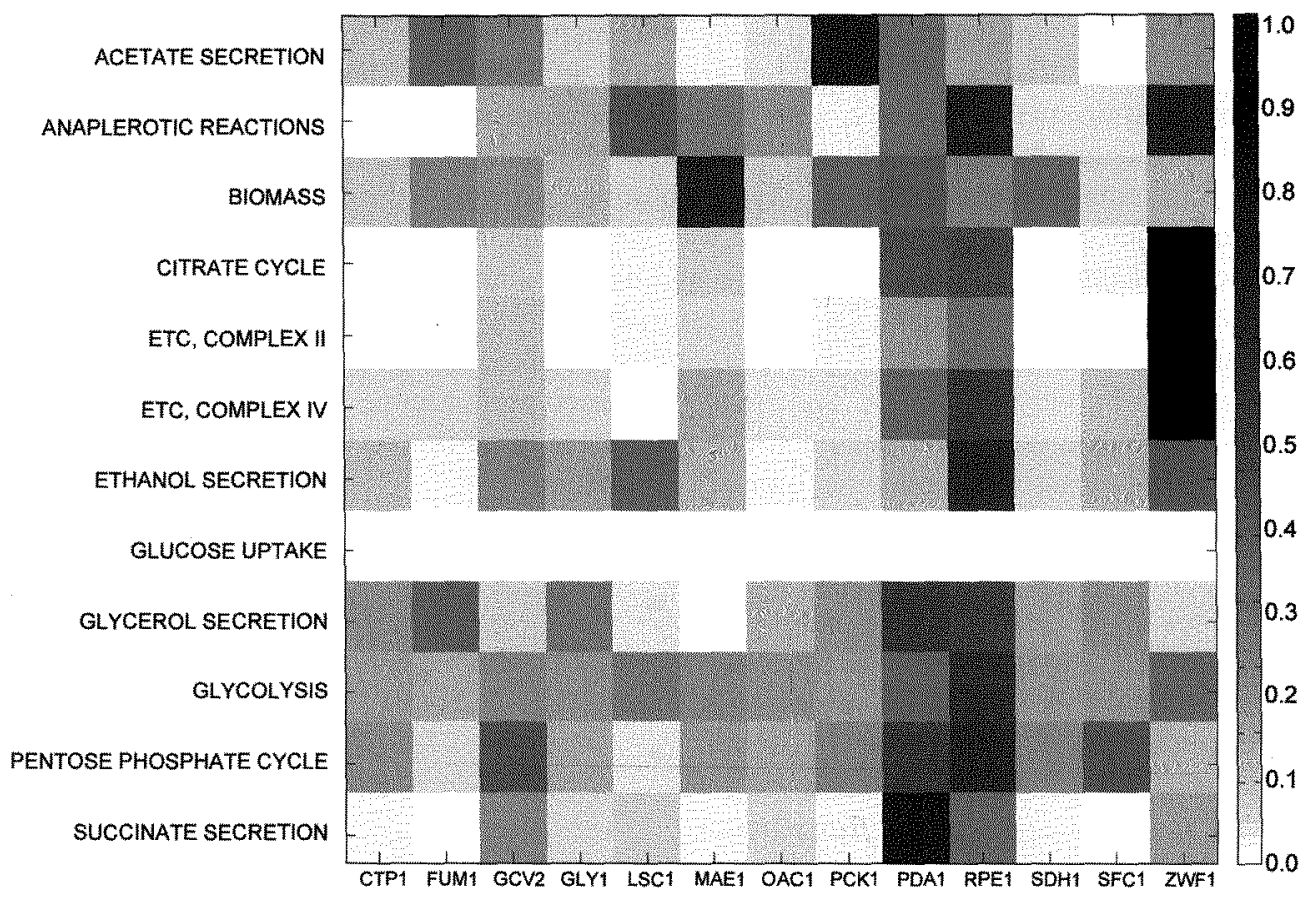

Fig. 3. Normalized difference of fluxes predicted by MOMA_LP_WT_CONSTR from experimental values.

Differences were computed between the experimentally determined and model predicted fluxes. Before taking the difference between fluxes, all fluxes were normalized by the glucose uptake rate for the given mutant. In order to make differences comparable for fluxes of different magnitudes, flux differences were then normalized by the range of a given flux across all experimental measurements. Finally, flux differences for reactions in the same metabolic pathway were averaged together to allow for easier interpretation of incorrect flux predictions. Displaying this data in a heatmap, where black represents maximal difference and white minimal difference, reveals that the largest differences between experimental and model predicted fluxes are for the $p d a l, z w f l$ and rpel mutants. This fits with correlation analysis, as these mutants had three of the lowest Spearman $\mathrm{R}$ values for the MOMA_LP_WT_CONSTR objective. Looking at the heatmap to identify the processes with the largest differences for these three mutants provides insight into the cause of the low correlations. For pdal, the large difference in succinate secretion is a result of the model failing to predict that the TCA cycle is used to maintain NADH/NAD balance in the absence of the pyruvate dehydrogenase reaction. For rpel, the model did not capture rerouting present in many pathways. Most of these reroutings stemmed from differential use of the pentose phosphate pathway resulting from the gene deletion. Finally, for $z w f$, there is a large increase in the flux through malic enzyme to compensate for the inability to produce NADPH through the pentose phosphate pathway. The increased flux through malic enzyme is associated with an increase in flux through the TCA cycle and the respiratory chain, which is not predicted by the model. In general, these three gene deletions all result in reroutings to maintain redox balance, and the full scope of these reroutings are missed by the model predictions. 
While the objective function which minimizes distance from an experimentally constrained wildtype solution was best for all mutants, there is variability in its relative performance across mutants. To explore this variability in more detail, we examined predicted fluxes for MOMA_LP_WT_CONTR, and assessed how well the fluxes though different metabolic pathways were predicted for different mutants. We hoped that the results of this analysis, which are displayed in a heatmap in Fig. 3, would provide insight into the sources of the decreased performance in certain mutants. The most erroneous flux predictions for most pathways are largely restricted to three mutants: rpel, pdal, and $z w f 1$. The pdal and $z w f l$ mutants are in reactions which utilize redox cofactors, and as described by Blank et al. such mutants tend to enact more distant rerouting to maintain redox balance. Therefore, it fits with intuition that using an objective function which minimizes distance from the wildtype would struggle in capturing more distant flux changes. A detailed examination of the predicted fluxes for these two mutants shows that while adjustments are predicted which resolve the redox imbalances caused by the given mutation, they are not the same adjustments found experimentally. For instance, for the pdal mutant, the NAD/NADH imbalance caused by the mutation is predicted to be resolved using the NADH dependant acetaldehyde dehydrogenase, but it seems that instead yeast increases respiratory activity to achieve redox balance. For the zwfl mutant, the model fails to predict the huge increase through the TCA cycle and malic enzyme, which occurs in yeast to counteract the deficiency in NADPH resulting from the lack of an intact pentose phosphate pathway. These examples indicate that the flux rerouting in yeast metabolism which takes place in order to maintain redox balance does not represent a minimal adjustment, or at least not minimal with respect to the distance metrics evaluated here.

\subsection{Prediction of absolute flux changes}

While the correlations computed above quantify how well the different objective functions predict the nature of flux reroutings in the various deletion mutants, they do not capture how well the different objectives predict the absolute flux through the system. As discussed above, while the 13 different mutants analyzed here largely have the same relative flux through different pathways as observed in the wildtype, the absolute flux varies greatly. To evaluate how well the different objective functions capture different mutations' effects on absolute flux, we compared predicted biomass production in each mutant to the corresponding experimentally measured values. The results of this comparison are displayed in Fig. 4 for the MOMA_LP_WT_CONSTR objective function. Fig. 4 indicates that despite the strong correlation between predicted and observed fluxes for all deletion mutants, there is little success in predicting the relative effects of the same mutations on the growth rate. The same trend observed in Fig. 4 was seen for all objective functions. Specifically, across all objective functions no mutant was predicted to have less than $90 \%$ of the wildtype growth, whereas experimental measurements found that 9 of the 13 mutants in fact had less than $90 \%$ of the wildtype growth rate. 


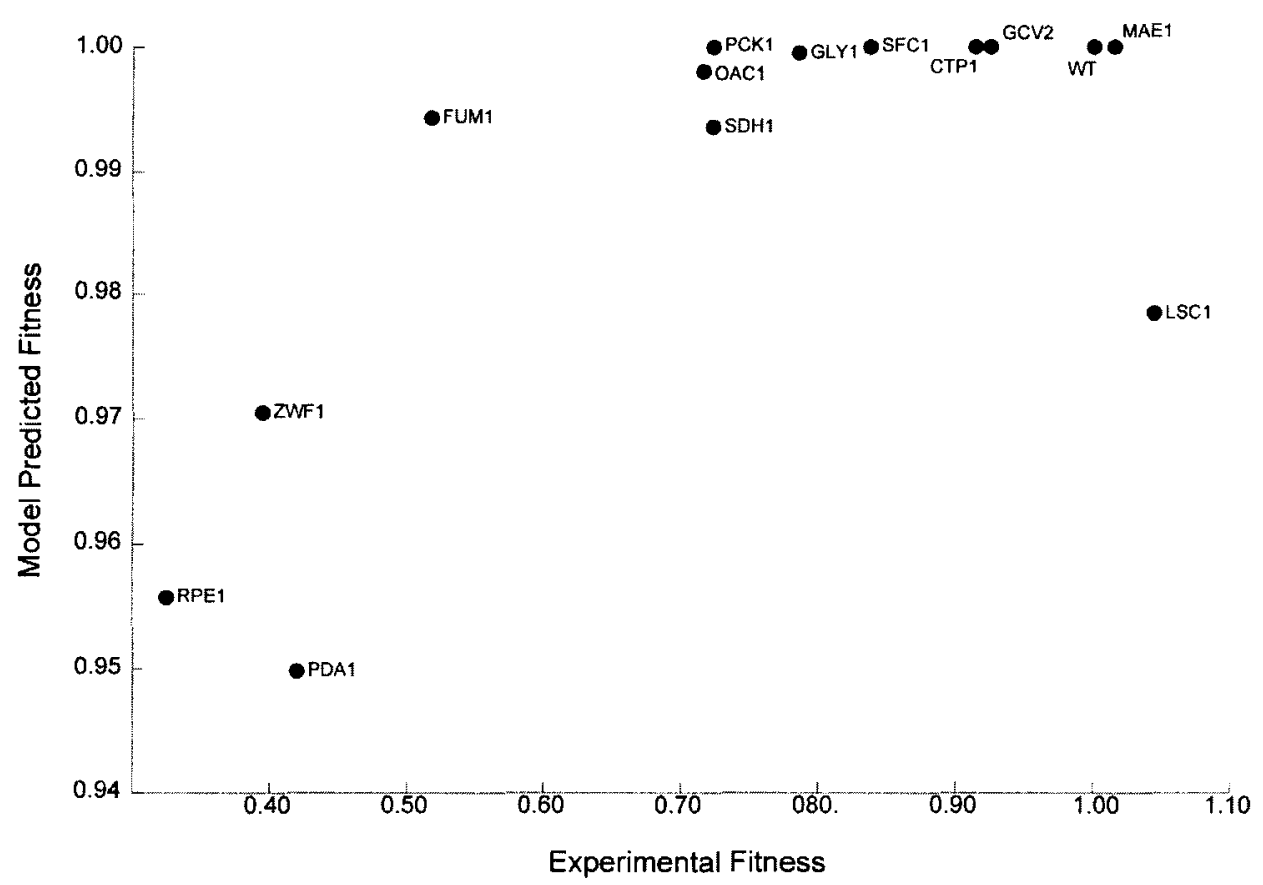

Fig. 4. Comparison of model predicted and experimentally determined growth rates for different strains.

Experimentally determined fitness was plotted against fitness predicted using the MOMA_LP_WT_CONSTR objective function for the 13 gene deletion and wildtype strains. Fitness was defined as the ratio between the growth rate of a given strain and the growth rate of the wildtype. While the experimental fitness values have a wide range across the 13 mutants, the model predicts that no mutant has a growth rate less than $95 \%$ that of the wildtype.

\section{Discussion}

We evaluated the proficiency with which yeast flux balance models can predict the flux response to a variety of gene deletion mutations. Specifically we assessed the flux predictions made by nine different objective functions, in response to 13 different single gene deletions. Comparison of flux predictions to complementary experimentally measured fluxes revealed that for all mutants the best performing objective functions were those which minimized the distance of mutant fluxes from an experimentally constrained wildtype solution. Importantly, while the 9 objective functions showed major differences in the accuracy of their predicted fluxes, all objectives correctly predicted that the 13 mutants would be able to produce biomass. This clearly demonstrates that the ability to correctly predict growth phenotypes does not necessarily translate into the ability to correctly characterize the underlying response at the level of reaction fluxes.

The fact that for all mutants the flux response was best described by objectives which implemented minimal flux rerouting, supports previous analyses of the metabolic response to gene deletions. Although the minimal rerouting objectives were consistently the best, predictions for all mutants were not equally good. Specifically, it was found that for mutants in reactions involving redox cofactors, a minimal adjustment was not 
sufficient to completely describe the flux response. We hypothesize that the reason for this is that there are a number of degrees of freedom in redox balancing, and the minimal rerouting criteria by itself is not sufficient to accurately predict the observed response. Likely, criteria which cannot easily be captured by flux balance models, such as enzyme affinity for redox substrates and kinetic rate constants, are crucial in determining how redox balance is achieved.

In addition to issues with redox mutants, all objective functions failed to predict the absolute flux for different mutants. Specifically, despite accurate predictions of how fluxes were rerouted in the mutants, the model predictions did not capture the reduction in the overall flux observed in the experiments.. The inability of any objective function to capture this aspect of the mutant response leaves the mechanism responsible for this observation unidentified. Again, it is likely that features of the metabolic response which cannot be captured by flux balance models are important here. Specifically, the relative efficiency of alternative pathways may limit the overall flux in mutants. Alternatively, regulatory responses to imbalances resulting from the gene deletions may cause an overall reduction in metabolic activity.

Despite some of the shortcomings in the abilities of flux balance models to predict mutant flux responses, overall they largely capture the salient features of the response to the different gene deletions. Importantly, the selection of objective function proved critical to the accuracy of the predicted fluxes, despite little effect on the prediction of mutant growth.

\section{Acknowledgements}

The authors would like to thank Bill Riehl and Hsuan-Chao Chiu for critical reading of the manuscript. The authors would also like to acknowledge support from the NASA Astrobiology Institute, the US Department of Energy, and Boston University.

\section{References}

[1] Blank, L.M., Kuepfer, L. and Sauer, U., Large-scale 13C-flux analysis reveals mechanistic principles of metabolic network robustness to null mutations in yeast, Genome Biol, 6(6):R49, 2005.

[2] Burgard, A.P., Pharkya, P. and Maranas, C.D., Optknock: a bilevel programming framework for identifying gene knockout strategies for microbial strain optimization, Biotechnol Bioeng, 84(6):647-57, 2003.

[3] Burgard, A.P., Nikolaev, E.V., Schilling, C.H., et al., Flux coupling analysis of genome-scale metabolic network reconstructions, Genome Res, 14(2):301-12, 2004.

[4] Deutscher, D., Meilijson, I., Kupiec, M., et al., Multiple knockout analysis of genetic robustness in the yeast metabolic network, Nat Genet, 38(9):993-8, 2006.

[5] Duarte, N.C., Becker, S.A., Jamshidi, N., et al., Global reconstruction of the human metabolic network based on genomic and bibliomic data, Proc Natl Acad Sci US A, 104(6):1777-82, 2007.

[6] Edwards, J.S., Ibarra, R.U. and Palsson, B.O., In silico predictions of Escherichia coli metabolic capabilities are consistent with experimental data, Nat Biotechnol, 19(2): 125-30, 2001. 
[7] Feist, A.M., Henry, C.S., Reed, J.L., et al., A genome-scale metabolic reconstruction for Escherichia coli K-12 MG1655 that accounts for 1260 ORFs and thermodynamic information, Mol Syst Biol, 3:121, 2007.

[8] Fischer, E. and Sauer, U., Large-scale in vivo flux analysis shows rigidity and suboptimal performance of Bacillus subtilis metabolism, Nat Genet, 37(6):636-40, 2005.

[9] Forster, J., Famili, I., Fu, P., et al., Genome-scale reconstruction of the Saccharomyces cerevisiae metabolic network, Genome Res, 13(2):244-53, 2003.

[10] Holzhutter, H.G., The principle of flux minimization and its application to estimate stationary fluxes in metabolic networks, Eur J Biochem, 271(14):2905-22, 2004.

[11] Johnston, M. and Kim, J.H., Glucose as a hormone: receptor-mediated glucose sensing in the yeast Saccharomyces cerevisiae, Biochem Soc Trans, 33(Pt 1):247$52,2005$.

[12] Kauffman, K.J., Prakash, P. and Edwards, J.S., Advances in flux balance analysis, Curr Opin Biotechnol, 14(5):491-6, 2003.

[13] Kuepfer, L., Sauer, U. and Blank, L.M., Metabolic functions of duplicate genes in Saccharomyces cerevisiae, Genome Res, 15(10):1421-30, 2005.

[14] Schilling, C.H., Covert, M.W., Famili, I., et al., Genome-scale metabolic model of Helicobacter pylori 26695, J Bacteriol, 184(16):4582-93, 2002.

[15] Schuetz, R., Kuepfer, L. and Sauer, U., Systematic evaluation of objective functions for predicting intracellular fluxes in Escherichia coli, Mol Syst Biol, 3:119, 2007.

[16] Segre, D., Vitkup, D. and Church, G.M., Analysis of optimality in natural and perturbed metabolic networks, Proc Natl Acad Sci U S A, 99(23):15112-7, 2002.

[17] Stolyar, S., Van Dien, S., Hillesland, K.L., et al., Metabolic modeling of a mutualistic microbial community, Mol Syst Biol, 3:92, 2007.

[18] Thomson, J.M., Gaucher, E.A., Burgan, M.F., et al., Resurrecting ancestral alcohol dehydrogenases from yeast, Nat Genet, 37(6):630-5, 2005.

[19] Vitkup, D., Kharchenko, P. and Wagner, A., Influence of metabolic network structure and function on enzyme evolution, Genome Biol, 7(5):R39, 2006. 